\title{
Progression of superficial venous insufficiency: analysis and implications for therapy
}

\author{
This article was published in the following Dove Press journal: \\ Journal of Vascular Diagnostics \\ 6 September 2013 \\ Number of times this article has been viewed
}

\author{
Vincenzo Puleo' \\ Pier Luigi Castagno ${ }^{2}$ \\ 'Servizio di Angiologia e Diagnostica \\ Vascolare, Torino, Italy; ${ }^{2}$ S.C. Chirurgia \\ Vascolare I, Azienda Ospedaliera Città \\ della Salute e della Scienza di Torino, \\ Torino, Italy
}

\begin{abstract}
Challenges to the widely accepted concept of retrograde progression of superficial venous insufficiency (SVI) have come from studies showing that the conventional descending paradigm is unable to account for many clinical presentations that would be better explained by the ascending hypothesis for the condition. Evidence substantiating this hypothesis holds therapeutic importance because, if confirmed, it would further weaken the rationale for aggressive surgery, in keeping with the general trend for alternative procedures. To evaluate the progression of SVI we performed echo Doppler examinations twice yearly for 5 years (ten examinations per patient) in 100 patients with SVI in which superficial venous branches (suprafascial) or segments of saphenous vein were incontinent, while the saphenofemoral junction was continent. Ascending progression of SVI was found in about $76 \%$ of patients. In the remaining patients, stabilization of the condition was probably due to its slow natural history. Venous insufficiency initially affecting the superficial venous branches of the leg variously progressed upward to involve the saphenous trunk in most cases and different sites in the vein, sometimes involving the saphenous ostium as well. The ascending hypothesis provides a convincing explanation for the various clinical presentations of SVI and the recurrence of varicosities after saphenectomy. In addition to intrinsic weakening of the superficial venous wall, elevated pressure in the calf venous circulation pushing on the superficial compartments, devoid of protective mechanisms, could be an alternative/complementary pathogenetic mechanism leading to structural weakening of the perforating vein valves. From a therapeutic perspective, the hypothesis for ascending progression of SVI is consistent with the use of alternative methods that halt the progression of venous disease and preserve the saphenous axis. Anatomical and functional assessment of ostial incontinence is key to achieving good outcomes after treatment. Of equal importance are the diameter and length of the incontinent saphenous trunk and the length of the continent saphenous segment below the contiguous incontinent saphenous segment. In cases of crossectomy, meticulous short or ultrashort stripping can maintain saphenous drainage through the perforators into the deep circulation.
\end{abstract}

Keywords: superficial venous insufficiency, hemodynamics, pathophysiology, surgery

\section{Introduction}

The conventional pathophysiological concept of superficial venous insufficiency (SVI) rests on the development of valve incompetence in an interfascial venous segment. In normal conditions, this segment connects the superficial to the deep venous systems, allowing the blood to flow from one to the other. According to this "descending" hypothesis, valve incompetence causes the blood to flow in the opposite direction, from the deep to the superficial systems, with retrograde propagation in the saphenous reservoir and the superficial collateral branches, leading to progression of the condition.
Correspondence: Vincenzo Puleo Servizio di Angiologia e Diagnostica Vascolare, ASL TOI, Via San Secondo 29 bis Torino, Italy Email vipuleo@tiscali.it 
Often, the interfascial segment identified as the cause is the saphenofemoral junction (SFJ); less often, it is the saphenopopliteal junction (SPJ). Altered function of a perforating vein may be the cause in remaining cases.

Although this theory is widely accepted, it fails to explain many of the clinical presentations of SVI encountered in routine practice. Its validity has been sporadically questioned, and alternative theories for the progression of SVI have been advanced. In a previous study that included examinations every 6 months for 2 years, we performed continuous-wave Doppler sonography in patients with a competent SFJ and with various stages of internal saphenous vein insufficiency. ${ }^{1}$ A review of imaging data from the past 20 years revealed that internal saphenous vein insufficiency will initially involve diverse sites distant from the SFJ, and that SFJ incompetence appears to occur secondary to progression of the condition. ${ }^{1}$ We have observed cases where insufficiency arose in a nonsaphenous vein, particularly in a superficial collateral tributary to the saphenous system, and where the saphenous trunk was secondarily involved through the tributary. However, what we found most striking was that SVI appeared to progress upwards.

Thiery, in a 1988 study describing the paraperoneal and soleus perforators, ${ }^{2}$ reported that a hallmark of insufficiency in these veins is that the "varices represent a symptom indicating a snag in the venous return on the anterior or posterior aspect of the thigh." This observation laid the groundwork for postulation of the "ascending" hypothesis for the progression of SVI, which became possible only after echo Doppler sonography entered routine clinical practice. Doppler imaging allowed for detailed visual documentation of changes over time and characterization of the variety of clinical presentations, starting from the earliest stages of the condition.

Except in cases that likely stabilized in response to prophylactic preventive treatment (compression, physical exercise, and medical therapy), the patterns of disease progression that emerged demonstrated worsening consistent with the ascending theory and confirmed doubts about the descending hypothesis. In the decade beginning in the late 1990s, a growing body of evidence supported the ascending theory; ${ }^{3-9}$ more recently, however, a less-sizable literature has become available, probably because echo Doppler examinations are routinely reserved for more severe cases of advanced-stage venous disease and because few studies have sufficiently long follow-up periods.

Motivated by the merits of the ascending hypothesis and encouraged by the renewed interest it has attracted, we thought it useful to report the results of a 5-year follow-up in patients with SVI.

\section{Materials and methods}

In the present study, 5-year follow-up data were collected for 145 limbs in 100 patients (age range 25-40 years) affected by SVI, with deep leg veins in the norm. Only the superficial collateral branches were incontinent, chiefly in the legs, with partial involvement of the saphenous trunk and competent SFJ. Hemodynamic studies, including echo Doppler examination (with the Paraná maneuver, the oscillation test, and the Valsalva maneuver), were performed every 6 months. This relatively young age range was specifically selected because, as emerged from an analysis of results from tests performed after our first study in 1992, progression of the condition was especially evident in patients in this age group. ${ }^{1}$ In most of the selected cases, incontinence developed in the posterior internal tributary in the leg; it was less often observed in the anterolateral or medial branches or the more posterior or anterior superficial branches. Less common were cases of an apparently incompetent segment initially in the internal saphenous vein of the leg, more often partial half to two thirds above, but nearly always confluent with an incontinent short superficial branch draining into an incompetent Cockett perforator. Furthermore, diverse presentations of venous incompetence were noted simultaneously in the same leg.

\section{Results}

During the 5-year follow-up, ascending progression of SVI could be clearly documented in most limbs (110/145; $75.8 \%$ ) as an initial site of venous reflux located in a segment upstream from the one detected on the previous control examination, whereas in other limbs $(35 / 145 ; 24.1 \%)$, progression was so unremarkable that it was interpreted as having stabilized. SVI evolved in various ways, involving diverse anatomical sites - some more often, others less often in relation to the location of the venous branch initially affected. This is consistent with the ascending hypothesis, although initial simultaneous involvement of more than one venous branch in the same limb was also noted.

In cases of incontinence of a posterior internal tributary of the leg, ascending valve incompetence often involved the internal saphenous trunk starting from the upper third of the leg, less often from the lower third of the thigh, at the confluence of this superficial collateral with the saphenous vein. An anterolateral branch of the leg usually involved the internal saphenous trunk of the middle-upper third of the leg, sometimes progressing anteriorly in the thigh to involve the 
anterior saphenous vein. Entering this short saphenous trunk might also be an initial lateral branch of the leg, which could communicate with the internal saphenous trunk or, more often, continue at the superficial level to the lateral or posterior aspect of the thigh, until reaching the root. It should be remembered that ascending progression of SVI can manifest itself in various and sometimes even unusual ways.

More defined and perhaps more easily classifiable were the final superficial sites involved in ascending progression of SVI. We noted that, in cases where the saphenous trunk was involved at various levels, the SFJ became incompetent at the so-called preterminal valve. In such cases, valve incompetence was noted to progress from the saphenous vein of the thigh to a superficial internal branch confluent with the pudendal system, to involve one of the saphenous collaterals at the SFJ before the valve, or to terminate at a Dodd's perforator at or above the middle third of the thigh, thus leading to initial incontinence of a Dodd's perforator. ${ }^{10}$ The SFJ can thus become incompetent in cases of anterior saphenous incontinence described above, manifesting as an incontinent SFJ but with reflux evident in only the anterior, not the internal, saphenous vein.
The terminal level of superficial ascending progression was also observed in the thigh perforators, including the perforator of the deep femoral vein or a Hunterian perforator (posterior aspect of thigh), particularly in cases of initial incompetence in the posterolateral or posteromedial branches in the leg. SVI might end before ascending in a perforator of the popliteal fossa or continue its ascent up to the root of the thigh in the gluteal or groin perforators. Rarely, we observed cases of SVI limited to the leg at the terminal ascending level, generally involving a high paratibial perforator, which is the escape point for superficial reflux (Table 1).

\section{Discussion}

Our findings show that, starting from venous reflux in distal branches of the lower limb, prevalently involving the superficial (extrasaphenous) branches of the leg, the initial site of reflux gradually ascends (in a centripetal direction), with extension of the refluent segment, eventually leading to valve incontinence in an interfascial segment (junction or perforator), which will become the so-called escape point in SVI.

We also observed that as SVI progresses, the perforators may occasionally become either the final site of ascending

Table I Clinical presentation of progression of varicose vein disease in 145 limbs

\begin{tabular}{|c|c|c|}
\hline $\begin{array}{l}\text { Initial incontinent segment } \\
\text { ( } 45 \text { limbs) }\end{array}$ & Ascending progression ( 1 I 0 limbs) & $\begin{array}{l}\text { Final point at surface } \\
\text { (82 limbs) }\end{array}$ \\
\hline \multirow{5}{*}{$\begin{array}{l}\text { Posterointernal superficial collateral } \\
\text { of leg }(67 \%)\end{array}$} & Internal saphenous trunk, upper third of leg (43\%) & SFJ $(72 \%)$ \\
\hline & Internal saphenous trunk, lower third of thigh (22\%) & Dodd's perforator (4\%) \\
\hline & Internal saphenous trunk, middle third of leg (I3\%) & Collateral of SFJ (I8\%) \\
\hline & External saphenous trunk, upper third of leg (6\%) & $\mathrm{SPJ}(7 \%)$ \\
\hline & Medial superficial collateral of thigh (16\%) & Perineal perforator (8\%) \\
\hline \multirow[t]{5}{*}{ Medial superficial collateral of leg (24\%) } & Internal saphenous trunk, upper third of leg (50\%) & SFJ $(60 \%)$ \\
\hline & Internal saphenous trunk, middle third of thigh (I2\%) & Dodd's perforator (5\%) \\
\hline & Internal saphenous trunk, lower third of thigh (38\%) & Collateral of SFJ (20\%) \\
\hline & & Internal branch of thigh - \\
\hline & & perineal perforator (I5\%) \\
\hline \multirow{5}{*}{$\begin{array}{l}\text { Anterolateral superficial collateral } \\
\text { of leg }(27 \%)\end{array}$} & Internal saphenous trunk of thigh (30\%) & SFJ $(56 \%)$ \\
\hline & Anterior saphenous trunk (10\%) & Collateral of SFJ (24\%) \\
\hline & Internal saphenous trunk, upper third of thigh $(60 \%)$ & Dodd's perforator (8\%) \\
\hline & & Internal branch of thigh - \\
\hline & & perineal perforator (I $2 \%)$ \\
\hline \multirow{4}{*}{$\begin{array}{l}\text { Short internal branch of thigh with internal } \\
\text { saphenous segment of leg (18\%) }\end{array}$} & Internal saphenous trunk of thigh (38\%) & SFJ $(50 \%)$ \\
\hline & Medial superficial collateral of thigh (36\%) & Collateral of SFJ (20\%) \\
\hline & Anterior superficial collateral of thigh $(24 \%)$ & Anterior saphenous - SFJ (I8\%) \\
\hline & & Perineal perforator (12\%) \\
\hline \multirow[t]{4}{*}{ Lateral superficial collateral of leg $(22 \%)$} & Anterior collateral of thigh - anterior saphenous trunk (27\%) & SFJ $(67 \%)$ \\
\hline & Anterior collateral of thigh - internal saphenous trunk (40\%) & Collateral of SFJ (16\%) \\
\hline & Lateral collateral of thigh $(20 \%)$ & Deep femoral perforator (I I\%) \\
\hline & Posterior collateral of thigh (I3\%) & Gluteal perforator $(6 \%)$ \\
\hline \multirow[t]{4}{*}{ Posterior superficial collateral of leg (20\%) } & External saphenous trunk (34\%) & SPJ $(38 \%)$ \\
\hline & Giacomini’s vein (I I\%) & Deep femoral perforator (I4\%) \\
\hline & Posterointernal collateral of thigh - internal saphenous & SFJ $(52 \%)$ \\
\hline & trunk $(55 \%)$ & Gluteal perforator (I2\%) \\
\hline
\end{tabular}

Abbreviations: SFJ, saphenofemoral junction; SPJ, saphenopopliteal junction. 
progression of valve incompetence or may have a predominant role in the pathogenesis of diverse presentations of SVI when they constitute, as frequently happens, the initial site of valve incompetence, which will ultimately involve the superficial segment contiguous with it. Thus, such veins would play the key pathogenic role once attributed to them. But in these circumstances, the perforator where initial incompetence begins will most often be found in the leg, whereas the escape-point perforator will more often be a thigh perforator.

The ascending hypothesis for progression of SVI may thus offer a more elegant and effective explanation for the various clinical presentations of varicose disease than does the classic descending theory, as it provides a better framework for understanding the centripetal progression of the underlying morphofunctional alterations in the venous system. Varicose recurrence after saphenectomy is yet another clinical presentation that fits this context, and our observations suggest that this recurrence develops in an ascending fashion. In this setting, varicose recurrence is due to elimination of the main superficial segment of venous drainage of the superficial circulation (the internal saphenous vein), thus creating the conditions for functional overload of the remaining superficial segments (superficial collateral branches), which, in turn, must compensate for the lack of the internal saphenous vein in its principal function as reservoir for draining blood from the superficial to the deep circulation. Overloading of these venous branches leads to dilatation and valve incompetence, ultimately resulting in varicosities (draining varicose veins) produced by their anatomic inadequacy in this new function, given that, unlike the saphenous trunks and deep veins, they are no longer protected by the fascia or muscles and therefore become incompetent.

This goes some way toward explaining the numerous occurrences of recurrent varicosity without a new SFJ. When encountered, however, a new SFJ, as hypothesized by several authors, ${ }^{11-13}$ may be consequent to a sort of neoangiogenesis that is frequently seen after vessel resection (ie, in the internal saphenous vein near the femoral vein). We believe, however, that this should not be interpreted as the origin of a new varicose formation but rather that it simply appears as such, when at a certain point a recurrent reflux varicose vein joins it as it progresses in ascending fashion in the attempt to reconnect to a vicarious superficial circulation.

Something similar occurs in the formation of varicose veins during pregnancy. Also in this instance, the varicose veins as they develop according to the ascending theory, may join together with other varicose formations of the leg originating from the groin and perineal regions. In this case, however, the latter represent an abnormal manifestation related to pelvic shunt (venous circuit connecting the pelvic veins and the veins of the lower limbs), because the pelvic varicocele that develops during pregnancy is characterized by varicose veins of the ovarian and uterine veins, an expression of pelvic congestion typical of pregnancy. The pressure gradient that causes these venous ectasias most likely pushes downward through these veins, leading to dilatation (varicosity) of the superficial veins of the root of thigh through the escape points resulting from incontinent pudendal and groin perforators. This condition probably represents the sole exception of formation of leg varices, exclusively due to the development of valve incontinence progressing in descending fashion.

We also found that the perforators in a setting of this type of development of SVI - when they often represent the final point of ascending progression of valve incontinence, thus becoming so-called escape points of superficial venous reflux - also appear to assume a prevalent role in the pathogenesis of various presentations of SVI when they represent, as they typically do, the initial point of valve incompetence that subsequently progresses to the contiguous superficial segment. In this way, these veins play the important pathogenetic role with which they were once attributed. In this instance, the perforator, the initial site of incompetence, will often be located in the leg, whereas the escape-point perforator will often be found in the thigh.

Although this pathophysiological concept may explain the natural history of the various presentations of SVI, it does not account for the underlying mechanism. A pathogenetic hypothesis recently proposed by Pittaluga et al, ${ }^{9}$ based on that advanced by Labropoulos et al, ${ }^{3,4}$ identified the intrinsic weakness of the vein wall of the superficial venous segments, specifically the suprafascia, as the cause of gradual dilatation with subsequent loss of valve continence. An alternative or complementary explanation for venous wall weakening is the development of hypertensive states in the venous circuits of the calf. ${ }^{14}$ The action of skeletal muscles, particularly the calf muscles, is considered to be the most important among hemodynamic factors. At distal sites where hydraulic resistance and hydrostatic pressure are greatest, the calf muscles have higher energy potential to compress the veins. This simple observation may explain why very high pressures can be found in the calf.

The mechanism of action of the muscle pump is similar to that of a volumetric pump, in which each cycle pushes downstream a quantity of fluid equal to the volume of the internal chamber and then aspirates the fluid upstream to refill the chamber, thus returning to the initial status. For this type 
of mechanism, the quantity of fluid pumped in circulation is determined solely by the size of the chamber (ie, the vein that is compressed) and the speed of compression. The pressure generated depends primarily on the hydraulic characteristics of the circuit in which the pump is working: the more the circuit is blocked (eg, by deep venous thrombosis), the higher the pressure the pump must generate to circulate the fluid. Hence, one can appreciate that in a calf where the pressure is already elevated, the pressure is augmented further in conditions such as those that lead to increased resistance of the abdominal veins (eg, in pregnancy or iliac vein stenosis). ${ }^{15}$ This pressure is transmitted to the vessel walls and valves.

With regard to the deep (subfascial) intermuscular and intramuscular compartments, protection by the muscles opposes vessel dilatation; the intrafascial saphenous segments are also protected, albeit less effectively. In the suprafascial compartments, where there is no such protection, the veins are more subject to dilatation if the pressure is not rebalanced. This difference in pressure between the more-internal and the more-external compartments can lead to tension on the perforator valves. If the walls rupture, this can cause structural weakening of the valve and render it incompetent.

Another possible mechanism we consider interesting and relevant in this discussion is fatigue weakening, which may occur even at pressures below the rupture threshold if tension is cyclical and repeated. At the incompetent valve, with each contraction of the muscle pump there is retrograde emptying through the external layers, with pressurization and consequent dilation of the superficial veins. Another consequence of pressurization of a superficial vein is the tendency of the pressurized fluid to empty through fissures in the superficial layer, where pressure is usually lower. When the fluid enters another vein, this vein, too, will be pressurized according to the principle of communicating veins. In serial fashion, the fluid will encounter the nearest vein and then, starting from the calf (hence, from below), involve adjacent areas in ascending progression.

\section{Therapeutic considerations}

Primary intervention at the SFJ and the internal saphenous vein, whatever the technique, is the most frequent procedure to interrupt or at least reduce reflux in the saphenous axis. In most cases, however, this is unwarranted according to the ascending or centripetal theory, which instead supports alternative methods that could halt the mechanisms underlying the progression of SVI. An attempt in this direction recently described by Pittaluga et $\mathrm{al}^{9,16}$ is an intervention that spares the refluxing internal saphenous vein and eliminates the incontinent superficial venous branches and the ectatic and tortuous (varicose) tributaries of the internal saphenous trunk - the same veins that, in keeping with the ascending theory, are the first to be affected before the potential involvement of the saphenous axis. The author states that this type of approach, called selective ablation of varices under local anesthesia (ASVAL) - basically a phlebectomy - was claimed to be effective in the midterm in the treatment of signs and symptoms of SVI, with abolition of internal saphenous vein reflux in more than two thirds of cases.

In our opinion, the positive results obtained with this treatment do not derive from the so-called varicose reservoir, which would presume an extremely dilated, tortuous, and incontinent superficial venous branch, but rather from components that play a role in the restoration of normal saphenous axis function and, more generally, the return of normal superficial drainage of the internal saphenous vein. These components include the reduction (fractioning) of the hydrostatic column via surgical disconnection of the superficial branch, with subsequently less pressure on the remaining incontinent saphenous segment, and abolition of the aspiration mechanism of the intrafascial saphenous axis by the contiguous part of the varicose superficial branch, closely related to the pressure gradient between the two segments. These components should produce important and long-lasting effects in restoration of saphenous trunk continence in conditions of reversibility, ie, when the source of the origin and the development of incontinence in the internal saphenous vein have been eliminated. Correct treatment of SVI should be able to halt the ascending progression of disease when reversibility of incontinence is still possible.

This may be obtained with the technique proposed by Pittaluga et $\mathrm{al}^{9,16}$ involving sclerotherapy and disconnection by high ligation of the incontinent superficial branch affluent of the internal saphenous vein, which is a type-2 CHIVA (conservative hemodynamic treatment for chronic venous insufficiency) as devised by Franceschi in $1988 .{ }^{17}$ To optimize the chances of creating a condition of reversibility of incontinence in the saphenous trunk and therefore a greater chance of success, planning for this type of treatment should take into account the following parameters and their predictive roles.

1. Normal function of the saphenofemoral ostium, defined as continence of the terminal valve, is a determinant factor for increasing the chance of therapeutic success.

2. In cases of an incontinent SFJ, it could be useful to (1) evaluate the degree of incontinence, investigating 
for so-called dissociated incontinence, ie, that occurring during a Valsalva maneuver but not during a compressionrelease test (aspiration-gravity test), as the dissociation is the expression of partial incontinence, and (2) evaluate reflux characteristics that, when not yet deep (slow velocity) although long, may be the expression of initial incontinence or incontinence of recent onset.

3. An incontinent saphenous vein diameter $<7 \mathrm{~mm}$ may suggest a good therapeutic outcome, although for this parameter one must also consider the close correlation between SFJ diameter and continence and their relationship with the contralateral saphenous vein having a normal diameter.

4. The length of the incontinent saphenous trunk, especially in the absence of complete ostial continence, affects the possibility of recovering continence in a shorter refluxing saphenous segment.

5. The length of the continent saphenous segment below the contiguous incontinent saphenous segment is an expression of effective propagation of anterograde flow in the saphenous vein.

The negative predictors of therapeutic outcome are various and include frank ostial incontinence with an incontinent saphenous segment $>8 \mathrm{~mm}$ in diameter and extending for more than two thirds of the thigh. In the presence of these clinical presentations, it is advisable - should ablative surgery be chosen - to minimize saphenous excision. To this end it may be useful to maintain efficient saphenous drainage into the deep circulation, exploiting the possibility of a reentry draining through a perforator, which eventually will substitute the saphenofemoral ostium at a lower level. These may be Dodd's perforators (Hunterian) or at least the upper paratibial perforators, allowing the option of short or ultrashort stripping above these veins. This, in our opinion, can contribute to reducing the risk of recurrence in such cases.

With regard to the pathogenesis of SVI, from a purely medical perspective, any therapeutic strategy that aims to block or counteract processes that may weaken the venous wall, which appears to be the fundamental factor underlying the development of SVI, is valid. The first step is to resolve, as practically as possible, the causes of hypertension in the deep venous circuit, particularly in the context of intra-abdominal veins. Recent studies on pharmacotherapy ${ }^{14}$ described the effect of sulodexide glycosaminglycans on enzymatic factors such as matrix metalloproteinases (MMPs), and found a direct correlation between the amount of sulodexide and modulation of the enzymatic activity of MMPs. Hyperactivity or overproduction of MMPs may lead to a reduction in the contractile ability of smooth muscle cells of the venous walls, with progressive venous dilatation.

\section{Disclosure}

The authors report no conflicts of interest in this work.

\section{References}

1. Castagno PL, Puleo V, Muncinelli M, Raso AM. Modalità di sviluppo dell'insufficienza della grande safena. Studio anatomico-statistico [Mode of development of the insufficiency of the great saphenous vein. Anatomical and statistical study]. Flebologia. 1992;3(4):389-393. Italian.

2. Thiery L. Le problème des perforantes [The problem with perforators]. Phlébologie. 1988;41(1):215-228. French.

3. Labropoulos N, Giannoukas AD, Delis K, et al. Where does venous reflux start? J Vasc Surg. 1997;26(5):736-742.

4. Labropoulos N, Leon L, Kwon S, et al. Study of the venous reflux progression. J Vasc Surg. 2005;41(2):291-295.

5. Engelhorn CA, Engelhorn AL, Cassou MF, Salles-Cunha SX. Patterns of saphenous reflux in women with primary varicose veins. $J$ Vasc Surg. 2005;41(4):645-651.

6. Caggiati A, Rosi C, Heyn R, Franceschini M, Acconcia MC. Age-related variations of varicose veins anatomy. J Vasc Surg. 2006;44(6): 1291-1295.

7. Bernardini E. L'evoluzione della malattia varicosa tronculare è ascendente o discendente? [Is the evolution of truncal varicose disease rising or falling?]. Acta Phlebologica. 2005;6:76-77. Italian.

8. Bernardini E, De Rango P, Piccioli R, et al. Development of primary superficial venous insufficiency: the ascending theory. Observational and hemodynamic data from a 9-year experience. Ann Vasc Surg. 2010;24(6):709-720.

9. Pittaluga P, Chastanet S, Réa B, Barbe R, Guex JJ. Nouveaux concepts hémodynamiques de l'insuffisance veineuse superficielle [New hemodynamic concepts of superficial venous insufficiency]. Phlébologie. 2008;61(1):61-69. French.

10. Puleo V, Castagno P. Vene Perforanti dell'arto Inferiore [Perforating Veins of the Lower Limb]. 2nd ed. Torino, Italy: Minerva Medica; 2008. Italian.

11. El Wajeh Y, Giannoukas AD, Gulliford CJ, Suvarna SK, Chan P. Saphenofemoral venous channels associated with recurrent varicose veins are not neovascular. Eur J Vasc Endovasc Surg. 2004;28(6): 590-594.

12. Winterbom RJ, Foy C, Earnshaw JJ. Causes of varicose vein recurrence: late results of a randomized controlled trial of stripping the long saphenous vein. J Vasc Surg. 2004;40(4):634-639.

13. Perrin MR, Labropoulos N, Leon LR. Presentation of the patient with recurrent varices after surgery (REVAS). J Vasc Surg. 2006; 43(2):327-334; discussion 334.

14. Mannello F, Raffetto JD. Le metalloproteinasi di matrice nella clinica della malattia venosa cronica [Matrix metalloproteinases in the chronic venous disease clinic]. Nautilus. 2012;1:10-16. Italian.

15. Raju S. Endovenous treatment of patients with iliac-caval venous obstruction. J Cardiovasc Surg (Torino). 2008;49(1):27-33.

16. Pittaluga P, Chastanet S, Réa B, Barbe R. Quelle est la place de la méthode ASVAL en 2008 [What is the place of the ASVAL method in 2008?]. Phlébologie. 2008;61(4):401-408. French.

17. Franceshi C, Cure CHIVA, 1988, Editions de L'Armancon, 21390 Precy-Sous-Thil France. French. 
Journal of Vascular Diagnostics

\section{Publish your work in this journal}

Journal of Vascular Diagnostics is an international, peer-reviewed journal of diagnostics, focusing on non invasive vascular investigation methods involved in the evaluation of vascular diseases. The journal is committed to the rapid publication in the fields of vascular diseases. Original research, review, case reports, expert opinion and 